\section{Military Technical College Kobry El-Kobba, Cairo, Egypt.}

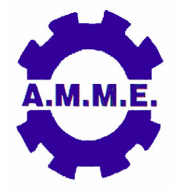

\title{
DYNAMIC MODELING AND CONTROL OF QUADROTOR VEHICLE
}

\author{
A. Y. Elruby ${ }^{\star}$, M. M. El-khatib**, N. H. El-Amary and A. I. Hashad ${ }^{++}$
}

\begin{abstract}
The control of Unmanned Aerial Vehicles (UAVs) is a very challenging field of research especially for Vertical Take-Off and Landing (VTOL) vehicles or aircrafts for their numerous advantages over the traditional airplanes and due to the rapid advances that were made in this field with the development of light weight MicroElectromechanical System (MEMS) sensors; it has become possible to build an autonomous model for a light weight quadrotor and to develop various controls for it. This paper focuses on the mathematical model of a quadrotor vehicle. A CAD model has been built for estimating mass and inertial properties of the physical model. Finally a PID controller for the proposed model is introduced then a Simulink model has been implemented for estimating the response of flight dynamics.
\end{abstract}

\section{KEY WORDS}

Modelling, PID control, quadrotor.

* Dept. of Mech. Mechatronics, AAST, Cairo, Egypt. Email: roubi12@hotmail.com.

** Egyptian Armed Forces. Email: elkhatib.m@gmail.com.

+ Dept. of Elec. Power, AAST, Cairo, Egypt. Email: noha helamary@hotmail.com.

++ Dept. of Comp. Eng., AAST, Cairo, Egypt. Email: hashad@cairo.aast.edu. 


\section{INTRODUCTION}

The quadrotor is a Vertical Take-Off and Landing (VTOL) aircraft [1] consists of 4 propellers arranged on $\mathrm{x}$-shape or +-shape. Every arm holds a propeller on its end as shown in Fig.1. The quadrotor has higher payload to total weight ratio compared to other aircrafts like single rotor and tandem rotor helicopters, simplicity of control and has a great manoeuvring attitude which can help in going into several areas cannot be accessed by traditional airplanes nor helicopters [2]. VTOL craft also offers direct access to buildings or areas making them a very fast form of transport between areas, especially those are elevated or have limited access.

The symmetry of the quadrotor body gives simplicity to the controller design as it can be controlled through varying the speed of the propellers [3]. Each two opposite propellers rotate in the same direction as shown in figure 1. The direction of rotation for each propeller reduces the mechanical complexity inherent in helicopters and other VTOL aircraft. Directional control is produced by individually altering the speed of the four motors. Collective pitch propellers are not required in a quadrotor design. This greatly reduces the mechanical complexity [4]. A quadrotor consists of two fixed pitch clockwise spinning propellers and two counter-clockwise spinning rotors which diagonally oppose each other as shown in Fig.1. This result that the reactive force of each propeller being effectively cancelled out by the diagonally opposite propeller's reactive component. This eliminates the need for a helicopter tail rotor.

Quadrotor craft has further efficiency and mechanical advantages. They have four small propellers as opposed to a single large propeller. Having small propellers reduces the torque on the system which means that the blades can be driven at higher velocities without producing additional mechanical vibrations and also increases motor efficiency. Less craft torque and vibration obviously put less stress on the mechanical components of the craft.

In this paper, a mathematical model for an under actuated six-degrees of freedom (6 DoF) quadrotor aircraft is derived based on Newton-Euler formalism. In addition the actuation forces are considered by modelling the aerodynamic forces and coefficients.

The model consists of two main parts, the first one is the translational system of equations and the second is the rotational system of equations. The quadrotor aircraft is a highly non-linear, Multi-Input Multi-Output (MIMO), strongly coupled and under actuated system with only four actuators [5]. This model is derived based on some assumptions in order to simplify the dynamics of that complex system to be suitable for simulation. These assumptions are as follows;

- The quadrotor structure is rigid and symmetric

- The propellers are rigid

- The ground effect is neglected 


\section{QUADROTOR SYSTEM MODEL}

The development of a suitable attitude controller for the quadrotor prototype required an accurate dynamic model to be developed. A Newtonian modelling method [2]was chosen to define the quadrotor dynamics for control purposes. The Newtonian method is the most popular choice for modelling rigid bodies in six degrees of freedom and has been used extensively for the modelling of traditional helicopters [2, $6]$. The derived model consists of 6 equations for the system dynamics and 4 equations describing the inputs of the system [1]. The dynamic equations are summarized as follows:

$$
\begin{aligned}
& \ddot{X}=(\cos \phi \sin \theta \cos \psi+\sin \phi \sin \psi) \frac{1}{m} U_{1}-1 / 2 C_{x} A_{x} \rho \dot{x}|\dot{x}| \\
& \ddot{y}=(\cos \phi \sin \theta \sin \psi-\sin \phi \cos \psi) \frac{1}{m} U_{1}-1 / 2 C_{y} A_{y} \rho \dot{y}|\dot{y}| \\
& \ddot{z}=(\cos \phi \cos \theta) \frac{1}{m} U_{1}-g-1 / 2 C_{z} A_{z} \rho \dot{z}|\dot{z}| \\
& \ddot{\phi}=\dot{\theta} \dot{\psi}\left(\frac{I_{y y}-I_{z z}}{l_{x x}}\right)+\frac{U_{z}}{l_{x x}} \\
& \ddot{\theta}=\dot{\phi} \dot{\psi}\left(\frac{l_{z z}-l_{x x}}{l_{y y}}\right)+\frac{U_{z}}{l_{y y}} \\
& \ddot{\psi}=\dot{\phi} \dot{\dot{\theta}}\left(\frac{l_{x x}-I_{y y}}{l_{z z}}\right)+\frac{U_{4}}{l_{z z}}
\end{aligned}
$$

The first 3 equations $(1,2$ and 3$)$ describes the linear acceleration of the vehicle in the direction of $x, y$, and $z$ axes respectively while the last 3 equations $(4,5$ and 6$)$ are nominated for the angular accelerations of the vehicle about the same axes respectively. " $\ell$ " represents the length of the arm holding the propeller, $(\phi, \theta, \psi)$ represent the Euler angles about the body axes $(x, y, z)$ respectively. $I_{x x}, I_{y y}$ and $I_{z z}$ are the inertial components about the $x$-axis, $y$-axis and $z$-axis respectively. Where $\mathrm{C}_{x^{\prime}} \mathrm{C}_{y}$ and $\mathrm{C}_{\mathrm{z}}$ are the translational drag coefficients, $\mathrm{A}_{x^{\prime}}, \mathrm{A}_{y}$ and $\mathrm{A}_{\mathrm{z}}$ are the propeller disk areas while $\dot{x}, \dot{y}$ and $\dot{z}$ are the translational velocity components along the main axes. Finally, $\rho$ is the air density.

Considering $\mathrm{U}_{\mathrm{i}}\left(\mathrm{i} \equiv 1_{3}, 2,3,4\right)$ to represent the input of the system, the following equations can be given [1];

$$
\begin{aligned}
& \mathrm{U}_{1}=\sum_{i=1}^{4} \mathrm{~T}_{\mathrm{i}}=\mathrm{b}\left(\Omega_{1}{ }^{2}+\Omega_{2}{ }^{2}+\Omega_{3}{ }^{2}+\Omega_{4}{ }^{2}\right) \\
& \mathrm{U}_{2}=\left(-\mathrm{T}_{2}+\mathrm{T}_{4}\right)=\mathrm{bl}\left(-\Omega_{2}{ }^{2}+\Omega_{4}{ }^{2}\right) \\
& \mathrm{U}_{3}=\left(\mathrm{T}_{1}-\mathrm{T}_{3}\right)=\mathrm{bl}\left(\Omega_{1}{ }^{2}-\Omega_{3}{ }^{2}\right)
\end{aligned}
$$




$$
\mathrm{U}_{4}=(-1)^{\mathrm{i}} \sum_{i=1}^{4} \mathrm{M}_{\mathrm{D}_{\mathrm{i}}}=\mathrm{d}\left(-\Omega_{\mathrm{i}}{ }^{2}+\Omega_{2}{ }^{2}-\Omega_{3}{ }^{2}+\Omega_{4}{ }^{2}\right)
$$

where $U_{1}$ represents the total thrust, $U_{2}$ represents the pitch moment, $U_{3}$ represents the roll moment, while $U_{4}$ represents the yaw moment. Finally, "b" is the thrust factor in hover and "d" is the drag factor in hover.

Increasing or decreasing of the speed of the four propellers together will be responsible for the altitude change in position and velocity while varying the speed of one pair of propellers $(\Omega 3$, and $\Omega 1)$ will cause the aircraft to tilt about the $y$-axis which is denoted as pitch angle theta " $\theta$ ". Similarly varying the speed of the propellers pair $(\Omega 4$, and $\Omega 2$ ) will cause the aircraft to tilt about $x$-axis which is denoted as roll angle phi " $\phi$ ".

Finally the vector summation of the reaction moment produced by the rotation of the pair $(\Omega 3$, and $\Omega 1)$ and the reaction moment produced by the rotation of the pair $(\Omega 4$, and $\Omega 2$ ) will cause the quadrotor to spin about its axis (z-axis) which is denoted as yaw angle epsi " $\Psi$ ". These are the six degrees of freedom of the system consisting of the position $(x, y, z)$ and the orientation $(\phi, \theta, \psi)$.

\section{COMPUTER AIDED DESIGN MODEL}

The CAD model was built up using SolidEdge (Synchronous Technology) ST in order to estimate the mass of the vehicle and its inertial properties about the main axes as to be used in the simulation as the system parameters. The assembly of the model is shown in Fig. 1 while the total estimated mass is equal to 688 grams and the inertial properties about the main axes $X, Y$, and $Z$ can be found in Table 1

\section{MATLAB/SIMULINK MODELLING}

A Matlab/simulink model was built using Matlab 2009 in order to simulate the systems dynamics and response under various conditions Fig. 3 shows the complete simulink model of the system

The model was built such that it has 4 inputs which are the desired altitude (position along the $Z$-axis) and the desired orientation of the vehicle consisting of the three Euler angles $\phi, \theta$ and $\psi$ about the axes $\mathrm{X}, \mathrm{Y}$ and $\mathrm{Z}$ respectively.

\section{CONTROLLER DESIGN AND TUNING}

Given time restraints, a PID controller was selected to provide attitude control for the quadrotor craft. 
The Euler angles are used as a feedback to the proposed controller in order to achieve the desired orientation. This may be used in the future with a path way trajectory controller which is not our concern in this study.

The traditional implementation can be defined as follows according to [7]:

$$
\text { Output }(\mathrm{t})=\mathrm{k}_{\mathrm{p}} \mathrm{e}(\mathrm{t})+\mathrm{k}_{\mathrm{i}} \int_{0}^{\mathrm{t}} \mathrm{e}(\mathrm{t}) \mathrm{dt}+\mathrm{k}_{\mathrm{d}} \frac{\mathrm{de}}{\mathrm{d} t}
$$

where $K_{p}$ is proportional gain, $K_{i}$ is integral gain, $K_{d}$ is derivative gain and $\mathrm{e}(\mathrm{t})$ is defined as the difference between the actual controlled variable value and a desired valued. The quadrotor controller will be targeted at forcing the attitude angles to desired set points. As a result $\mathrm{e}(\mathrm{t})$ can be defined as:

$$
\begin{aligned}
& \mathrm{e}_{\text {roll }}(\mathrm{t})=\text { Set point }- \text { Measured Roll } \\
& \mathrm{e}_{\text {pitch }}(\mathrm{t})=\text { Set point }- \text { Measured Pitch } \\
& \mathrm{e}_{\text {yaw }}(\mathrm{t})=\text { Set point }- \text { Measured Yaw }
\end{aligned}
$$

These are the attitude and altitude controllers. First, the attitude controller, it is shown in Fig.4. The controller has 3 inputs; the desired Euler angles and it takes the actual Euler angles values as a feedback and it calculates the appropriate control inputs (control command) $U_{2}$ which is responsible for the pitching moment, $U_{3}$ which is responsible for rolling moment and finally $U_{4}$ which is responsible for yawing moment.

The second is the altitude controller which is similar to the attitude controller but it only controls the position of the craft along the $z$ axis. It means that it only controls the altitude of the craft. It has the desired altitude as an input in addition to the actual altitude as a feedback signal while the output is the appropriate thrust force that keeps the craft at the desired altitude. Both controllers were manually tuned.

\section{STEP RESPONSE OF THE SYSTEM}

The system was tested for a step input to the desired altitude and the output is shown in Fig. 6. The system response is fast and has an acceptable overshoot about $12.3 \%$ in addition to small steady state error of $0.02 \%$. The system response for a step input to the desired phi was not as better as the response of the system to the desired altitude Fig.7 shows this response. The system reaches the desired phi after more than $200 \mathrm{sec}$ and with about $15 \%$ steady state error which is not acceptable. Theta response is similar to the phi response and this is due to the similarity in dynamic equations which came from the symmetry of the quadrotor structure. Another method of control can be applied for improving the system output. This can be done in future work.

\section{PULSE RESPONSE OF THE SYSTEM}

The system was tested for a pulse input of desired altitude equal to $5 \mathrm{~m}$ for $4 \mathrm{sec}$ and 
then it should reach the zero altitude again and Fig.8 shows the response of the system.

The response of the system for a desired phi pulse was not sufficient and it is not acceptable Fig. 9 shows the response of the system again it reaches the desired input after very long time about $20 \mathrm{sec}$ in addition to a very large over shoot about more than $300 \%$ percent of the input which is not acceptable.

\section{CONCLUSION}

The quadrotor model mathematical model was different than the other built models as the drag forces along the 3 axes is included, in addition to the coupling between the translation dynamics and the angular dynamics. The proposed PID controller used is sufficient and suitable for controlling the altitude (motion in Z-direction) of the quadrotor craft but it is not sufficient for controlling the attitude or Euler angles of the quadrotor craft. This suggests proposing an Artificial Intelligence (AI) controller in future work. The system equations of the model shows that the Euler angles dynamics is independent and it does not rely on the translational dynamics while the translational dynamics is dependent on the angular dynamics, this is clear from the equations of motion of translation as they are function of Phi, Theta, and Psi.

In future, an artificial intelligent controller may be introduced for the attitude controller of the same model in order to achieve a better performance.

The major drawback in the model is that the motor dynamics is not included which will be affecting the dynamics of system it can be added in the future work of this research.

\section{REFERENCES}

[1] V. MARTíNEZ, "Modelling of the Flight Dynamics of a Quadrotor Helicopter," in Aerospace Sciences. vol. Master of Science: Cranfield, 2007.

[2] K. P. Valavanis, Advances in Unmanned Aerial Vehicles vol. 33. Florida: Springer, 2007.

[3] T. Bresciani, "Modelling, Identification and Control of a Quadrotor Helicopter," in Automatic Control. vol. Master of Science Sweden: Lund, 2008.

[4] G. M. H. Haomiao Huang, Steven L. Waslander and Claire J. Tomlin, "Aerodynamics and Control of Autonomous Quadrotor Helicopters in Aggressive Maneuvering," in IEEE International Conference on Robotics and Automation Kobe, Japan, 2009.

[5] D.-b. W. Ashfaq Ahmad MIAN, "Dynamic modeling and nonlinear control strategy for an underactuated quad rotor rotorcraft," Zhejiang University SCIENCE, vol. 249, 2008.

[6] D. P. RAYMER, Aircraft Design: A Conceptual Approach. Washington: AIAA, 2004.

[7] M. M. Atheer L. Salih, Haider A. F. Mohamed and Khalaf Sallom Gaeid, "Modelling and PID Controller Design for a Quadrotor Unmanned Air Vehicle," in IEEE International Conference on Automation Quality and Testing Robotics (AQTR) Cluj-Napoca 2010. 


\section{Table and Figures:}

Table 1. Inertial properties of the system.

\begin{tabular}{|c|c|c|}
\hline Axis & $\begin{array}{c}\text { Mass Moment } \\
\text { Inertia, [kg.m }{ }^{2} \text { ] }\end{array}$ & $\begin{array}{c}\text { Radii of } \\
\text { Gyration, } \\
\text { [mm] }\end{array}$ \\
\hline $\mathrm{X}$ & 0.003325 & 88.19 \\
\hline $\mathrm{Y}$ & 0.003263 & 87.36 \\
\hline $\mathrm{Z}$ & 0.006430 & 122.64 \\
\hline
\end{tabular}

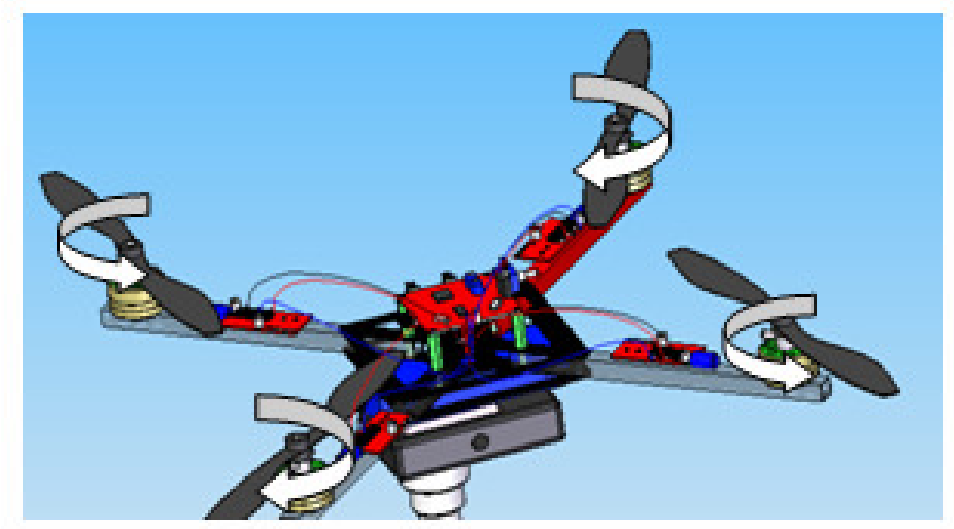

Fig.1. Quadrotor operation.

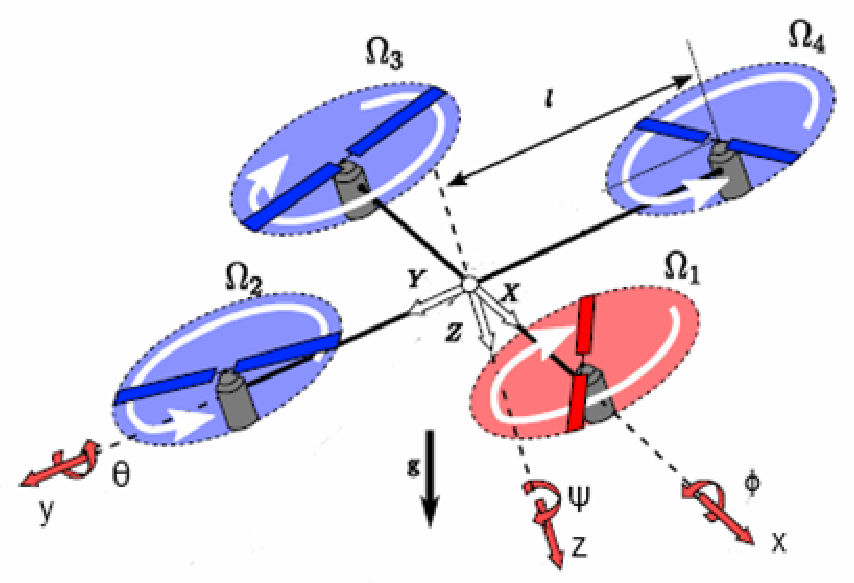

Fig. 2. Axes notation. 


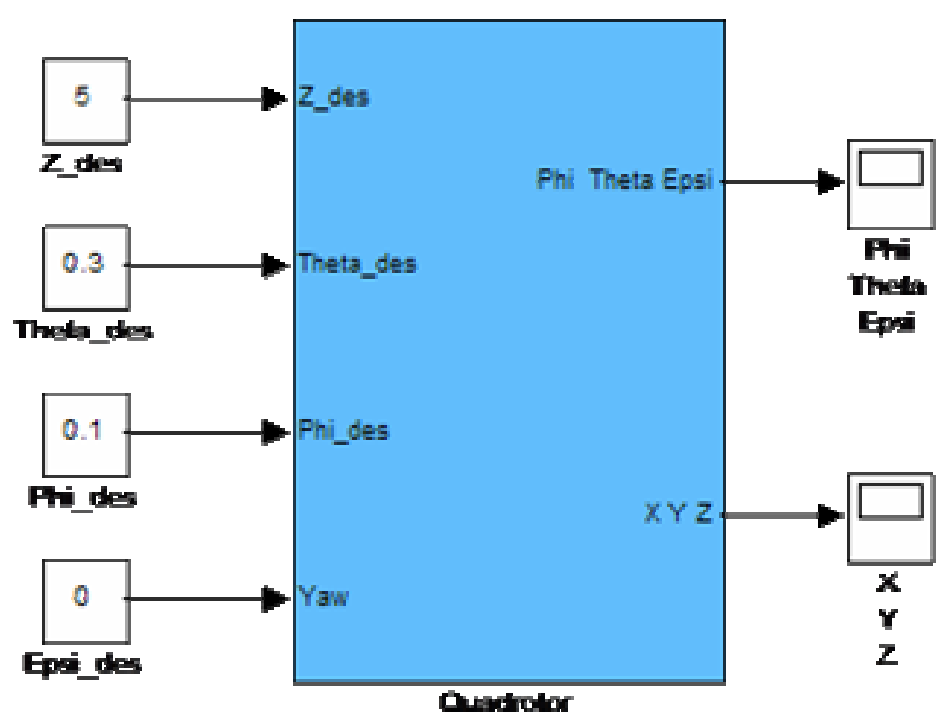

Fig.3. Simulink model.

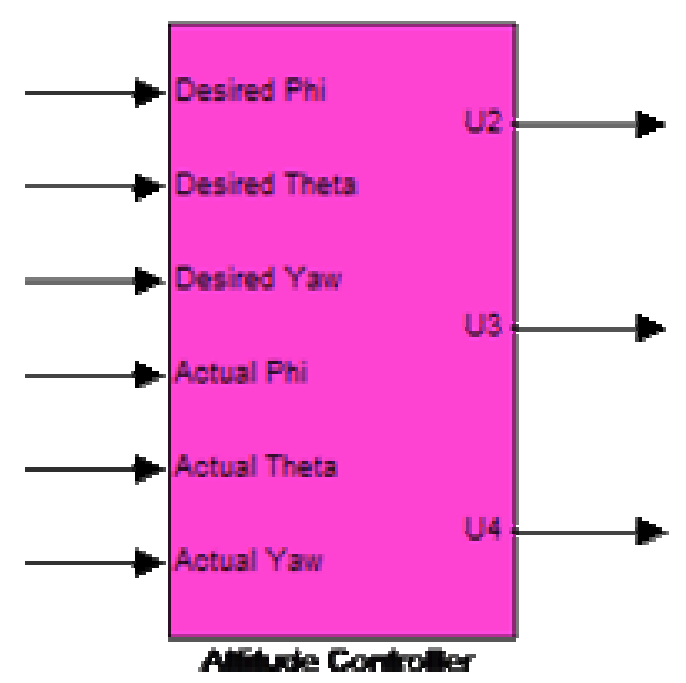

Fig.4. Attitude controller block.

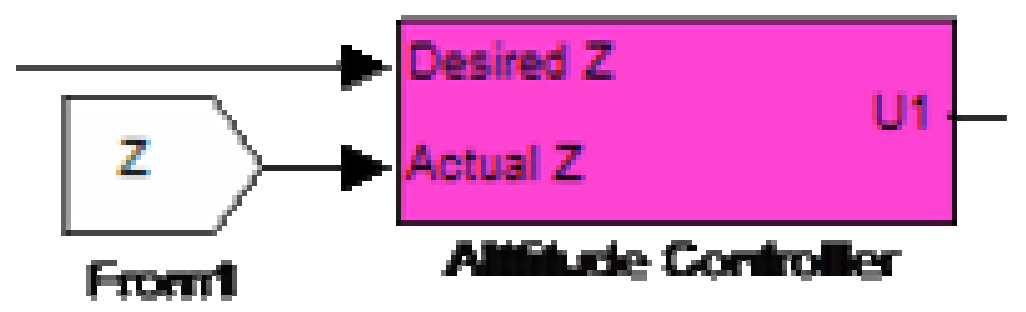

Fig.5. Altitude controller block. 


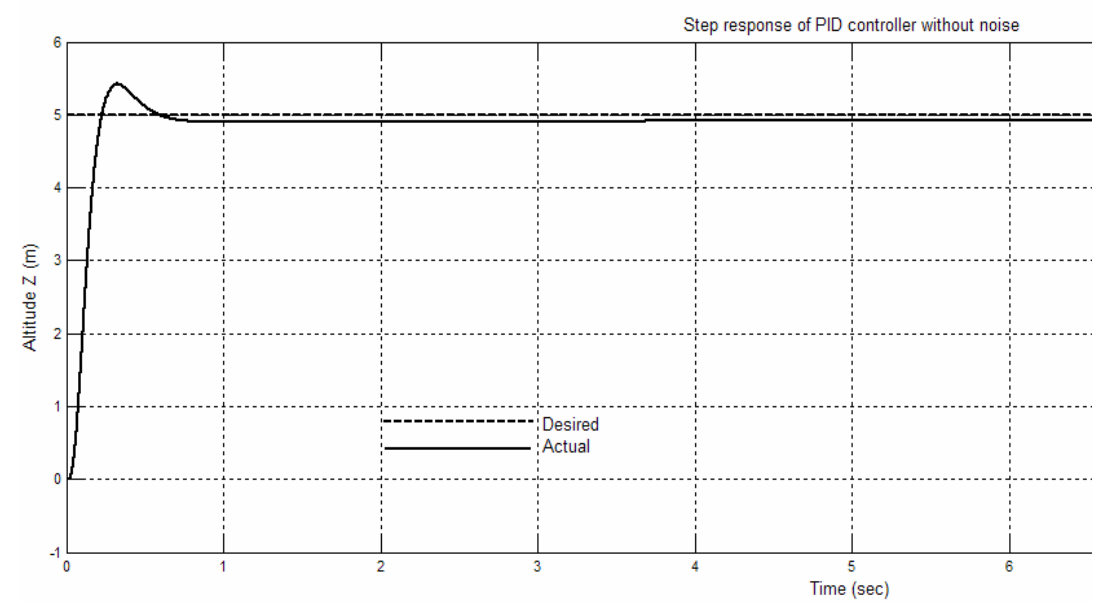

Fig.6. Step response of Z.

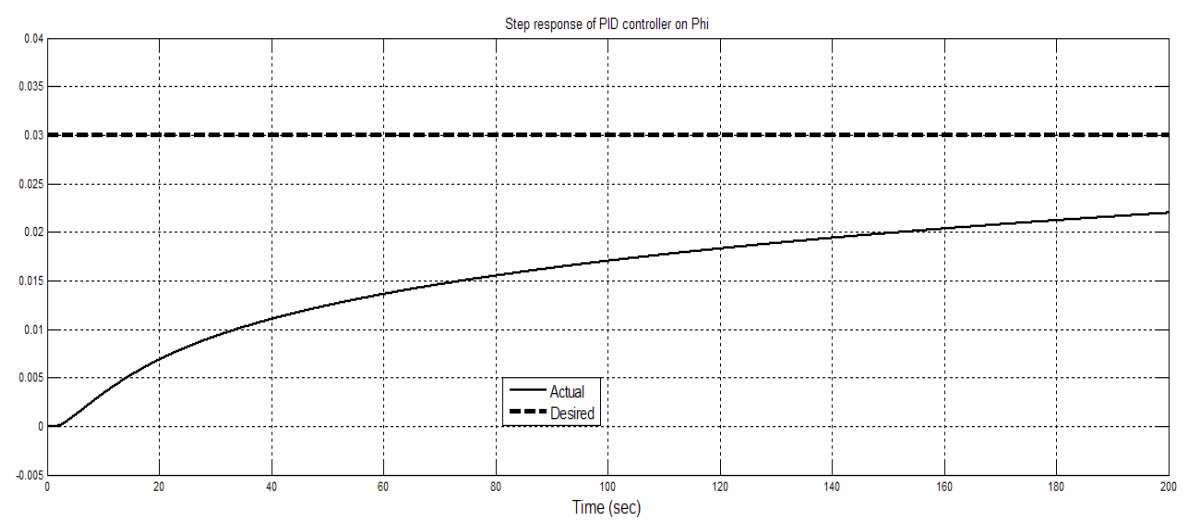

Fig.7. Step response of Phi.

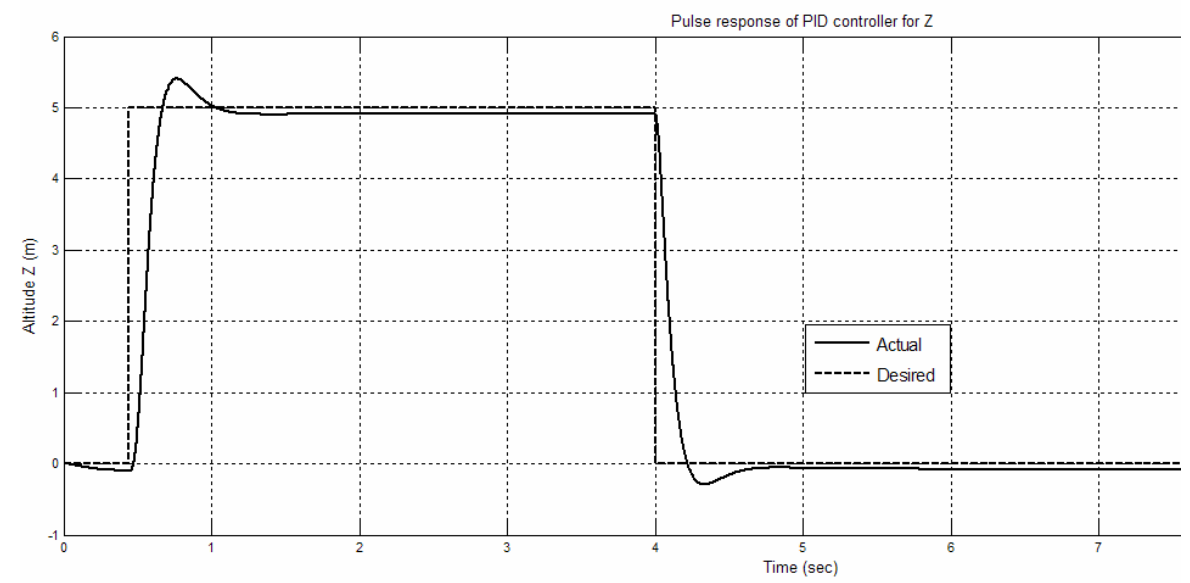

Fig.8. Pulse response of $Z$. 


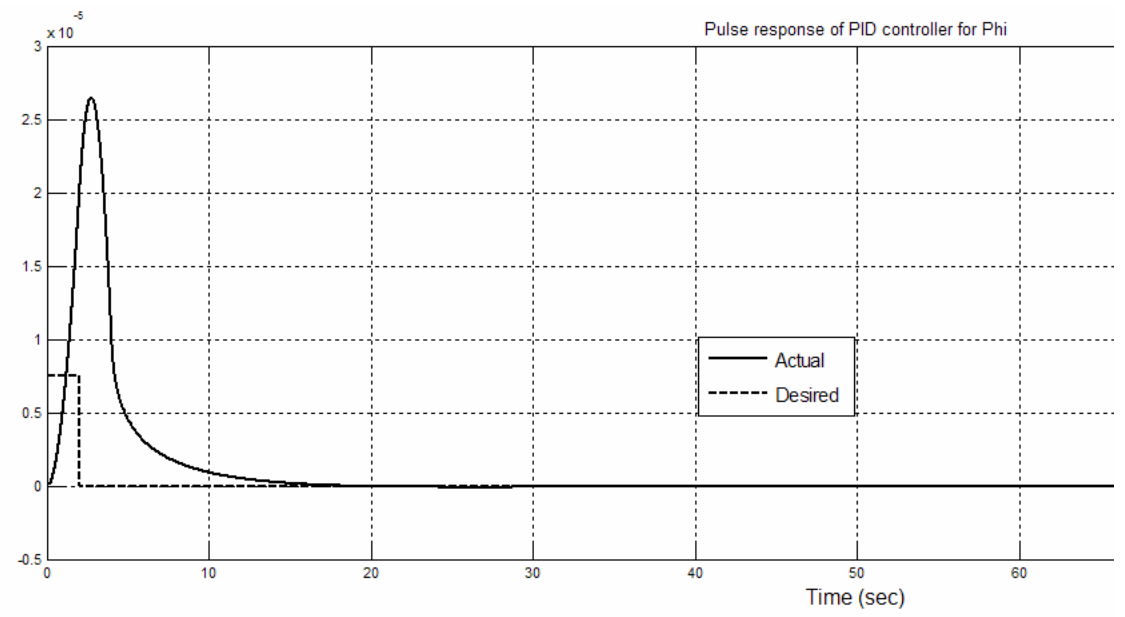

Fig.9. Pulse response of Phi. 